\title{
Evidence and implications for exciton dissociation in lead halide perovskites
}

\author{
Vandana Tiwari ${ }^{1,2}$, Hong-Guang Duan ${ }^{1,3,4}$, Ajay Jha ${ }^{1}$, Pabitra K. Nayak ${ }^{5}$, Michael \\ Thorwart $^{3,4}$, Henry J. Snaith ${ }^{5}$, and R. J. Dwayne Miller ${ }^{1,4,6, *}$ \\ ${ }^{1}$ Max Planck-Institute for the Structure and Dynamics of Matter, Luruper Chaussee 149, 22761 \\ Hamburg, Germany \\ ${ }^{2}$ Department of Chemistry, Universität Hamburg, Martin-Luther-King platz 6, Germany \\ ${ }^{3}$ I. Institut für Theoretische Physik, Universität Hamburg, Jungiusstraße 9, 20355 Hamburg, Germany \\ ${ }^{4}$ The Hamburg Center for Ultrafast Imaging, Luruper Chaussee 149, 22761 Hamburg, Germany \\ ${ }^{5}$ Clarendon Laboratory, Department of Physics, University of Oxford, Parks Road, Oxford OX1 3PU, \\ United Kingdom \\ ${ }^{6}$ The Departments of Chemistry and Physics, University of Toronto, 80 St. George Street, Toronto, \\ M5S 3H6 Canada
}

\begin{abstract}
We have employed ultrafast transient-grating and twodimensional electronic spectroscopy to probe dynamics of photo-excited $\mathrm{CH} 3 \mathrm{NH} 3 \mathrm{PbI} 3$ thin films with 16 -fs temporal resolution. We distinctly capture the 30-fs decay of excitons, weakly coupled to the phonons.
\end{abstract}

\section{Introduction}

Hybrid lead halide perovskites (LHPs) are an emergent class of materials for efficient optoelectronic devices owing to their facile solution processability and high charge carrier mobility [1-2]. With the advancement in fabrication methods, the power conversion efficiency of perovskite-based photovoltaics has progressed to $22.1 \%$ [3]. For further rational tailoring of the device efficiency, it is imperative to understand the fundamental process of photoinduced charge generation and their transport in perovskites. Savenije and co-workers have proposed a direct-indirect character of the bandgap in methylammonium lead iodide, $\mathrm{CH}_{3} \mathrm{NH}_{3} \mathrm{PbI}_{3}$ [4]. In addition, recent studies have proposed a significant role for excitons in this material, although there has been no conclusive report of the direct generation of excitons in this material. Therefore, a deeper understanding of the nature of primary excitation and the magnitude of coupling strength of the photo-generated species to phonons will pave the way towards a comprehensive knowledge of charge transfer and transport pathways in perovskites. Here, we use a combination of ultrafast transient grating and two-dimensional (2D) electronic spectroscopy to probe the primary excitation process along with the impulsively excited vibrational modes in $\mathrm{CH}_{3} \mathrm{NH}_{3} \mathrm{PbI}_{3}$ at room temperature and $180 \mathrm{~K}$. We distinctly capture the signature of excitons, which are weakly coupled to phonons. We also observe a coherent phonon at $\sim 150 \mathrm{~cm}^{-1}$, pertaining to methyl-libration, which is distinct to the excited state.

*Corresponding author: dwayne.miller@mpsd.mpg.de 


\section{Experimental Setup}

Ultrashort light pulses were generated by a home-built non-collinear optical parametric amplifier (NOPA) pumped by a commercial femtosecond laser Pharos (Light Conversion). The laser pulses were compressed to $16 \mathrm{fs}$ FWHM. All measurements have been performed in all-reflective 2D spectrometer [5]. The pulses are focused on the sample with a spot size of $100 \mu \mathrm{m}$, and the photon-echo signal is generated in the phase-matching direction. For transient grating measurements, the NOPA spectrum was centered at $12740 \mathrm{~cm}^{-1}$, while for two-dimensional electronic spectroscopy measurements, it was centered at $13300 \mathrm{~cm}^{-1}$ covering the band-gap and sub band-gap transitions (Fig. 1). To avoid oxidation damage, the sample was kept in a cryostat (MicrostatHe2, Rect. Tail from Oxford Instruments) under vacuum conditions $\left(\sim 10^{-6} \mathrm{~Pa}\right)$.

\section{Results and Discussion}

The 2D electronic spectrum of $\mathrm{CH}_{3} \mathrm{NH}_{3} \mathrm{PbI}_{3}$ thin films at ambient temperature is shown in Fig. 1. The frequency window of the laser pulse $\left(11500-14500 \mathrm{~cm}^{-1}\right)$ is tuned to cover the band-gap transition as well as the proposed excitonic-transition as shown in Fig. 1(a). The $2 \mathrm{D}$ spectrum at $\mathrm{T}=0 \mathrm{fs}$ in Fig. $1(\mathrm{~b})$, distinctly show an exciton peak at $\omega_{\tau}=13100 \mathrm{~cm}^{-1}$ in addition to the free carrier peak above $13500 \mathrm{~cm}^{-1}$. This finding gives direct spectroscopic evidence for the coexistence of excitons and free carriers in $\mathrm{CH}_{3} \mathrm{NH}_{3} \mathrm{PbI}_{3}$ at room temperature [6]. The observed value of the excitonic transition has been used to obtain the exciton binding energy, $E_{b}$ of $\sim 12 \mathrm{meV}$. Thus, the $E_{b}$ for tetragonal phase of hybrid perovskite retains a similar value to that determined at low temperatures for orthorhombic phase. Fitting the anti-diagonal linewidths in $2 \mathrm{D}$ electronic spectrum for $\mathrm{T}=0 \mathrm{fs}$ along the line cuts shown as A and B in Fig. 1(b) to Lorentzian line shapes provides the electronic dephasing timescales of $39 \pm 1.5$ and $41 \pm 1.5$ fs corresponding to the exciton and free carriers transitions, respectively. Interestingly, the dephasing timescales are comparable to values for small solvated dye molecules such as cyanine [7], which suggests that excitonphonon interactions in LHP may arise from similar deformation potentials. This also suggests that the excitons and electrons in LHP are protected by charge screening effects.
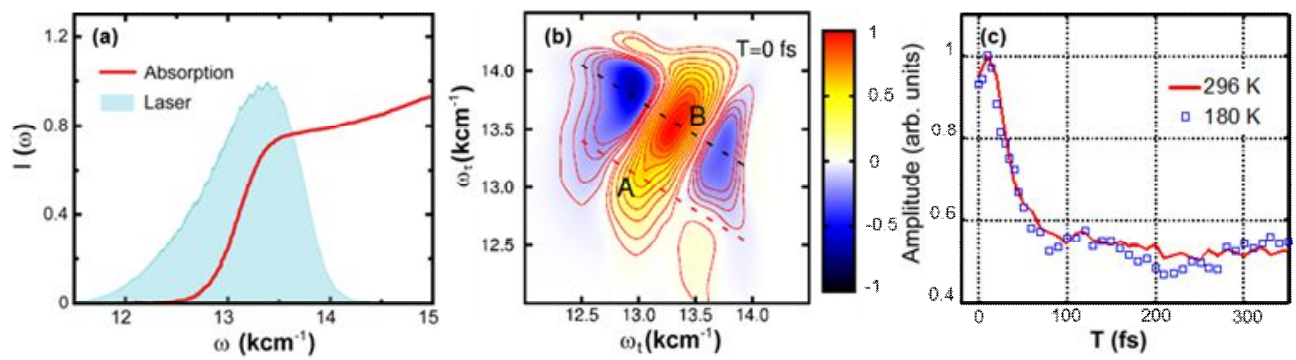

Fig. 1. Two-dimensional (2D) electronic spectroscopy: (a) Absorption spectrum of $\mathrm{CH}_{3} \mathrm{NH}_{3} \mathrm{PbI}_{3}$ thin film at room temperature shown as a red trace. Blue shaded curve represents the laser spectrum used for the measurements; (b) 2D electronic spectrum at waiting time $\mathrm{T}=0 \mathrm{fs}$; (c) exciton decay kinetics at different temperatures: $296 \mathrm{~K}$ (red line) and $180 \mathrm{~K}$ (blue square). Adapted with permission from reference [6]. Copyright (2017) American Chemical Society.

Additionally, the high temporal resolution of our set-up helps us to capture the decay of excitons with an $\sim 30$ fs time constant at room temperature and $180 \mathrm{~K}$, as shown in Fig. 
1(c). The rate of dissociation of excitons is insensitive to the temperature factor, which hints towards entropically driven exciton decay in LHP within the electronic dephasing timescales. Power spectra of vibrational modes at the cross-peak between exciton and freecarrier signatures, $\left(\omega_{\mathrm{t}}, \omega_{\tau}\right)=(13500,13100) \mathrm{cm}^{-1}$ shows a strong vibrational mode of $\sim 190$ $\mathrm{cm}^{-1}$, as shown in Fig. 2(a).
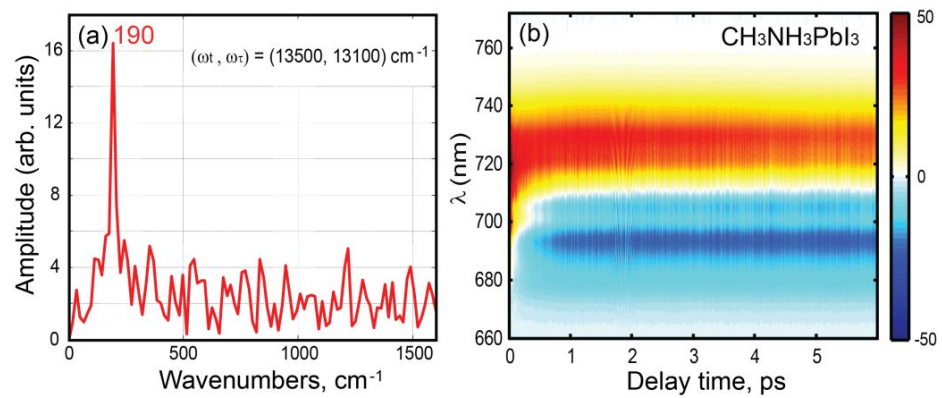

Fig. 2. (a) Power spectrum of vibrational modes at $\left(\omega_{t}, \omega_{\tau}\right)=(13500,13100) \mathrm{cm}^{-1}$. (b) Transient grating (TG) spectrum of $\mathrm{CH}_{3} \mathrm{NH}_{3} \mathrm{PbI}_{3}$ thin film at room temperature. Blue colour represents excited state absorption and red colour is ground state bleach.

To gather explicit understanding of ground and excited state vibrational modes, we have tuned our laser spectrum to centre at $12740 \mathrm{~cm}^{-1}$ and performed transient grating (TG) spectroscopy. Fig. 2(b) shows TG spectrum for $\mathrm{CH}_{3} \mathrm{NH}_{3} \mathrm{PbI}_{3}$ at room temperature with signatures of excited state absorption and ground state bleach. To extract the coherent wave packets of optical phonons coupled to the electronic transitions, Fourier analysis was performed on intensity modulated TG spectrum, by subtracting the background from the decay components at all probe wavelengths. We distinctly capture a coherently excited phonon at $\sim 150 \mathrm{~cm}^{-1}$ exclusive to the excited state. This mode of methyl-libration along with other phonon modes of inorganic octahedral might play a significant role in crystalline distortion upon carrier generation.

\section{References}

1. M.M. Lee, J. Teuscher, T. Miyasaka, T.N. Murakami, H.J. Snaith, Science 338, 643 (2012).

2. J. Burschka, N. Pellet, S.J. Moon, R. Humphry-Baker, P. Gao, M.K. Nazeeruddin, M. Grätzel, Nature 499, 316 (2013).

3. W.S. Yang, J.H. Noh, N.J. Jeon, Y.C. Kim, S. Ryu, J. Seo, S.I. Seok, Science 348, 1234 (2015).

4. E.M. Hutter, M.C. Gélvez-Rueda, A. Osherov, V. Bulović, F.C. Grozema, S.D. Stranks, T.J. Savenije, Nat. Mater. 16, 115 (2017).

5. V.I. Prokhorenko, A. Picchiotti, S. Maneshi, R.J.D. Miller, Springer Proc. Phys. 162, 432 (2015).

6. A. Jha, H.G. Duan, V. Tiwari, P.K. Nayak, H.J. Snaith, M. Thorwart, R.J.D. Miller, ACS Photonics 5, 852 (2017).

7. A. Halpin, P.J.M. Johnson, R. Tempelaar, R.S. Murphy, J. Knoester, T.L.C. Jansen, R.J.D. Miller, Nat. Chem. 6, 196 (2014). 